\title{
Intraoperative Seizures Detected as Increased Bispectral Index Values during Posterior Fossa Surgeries
}

\author{
Deepti Srinivas ${ }^{1}$ Muthuchellappan Radhakrishnan ${ }^{1}$ \\ Manohar Nitin ${ }^{2}$

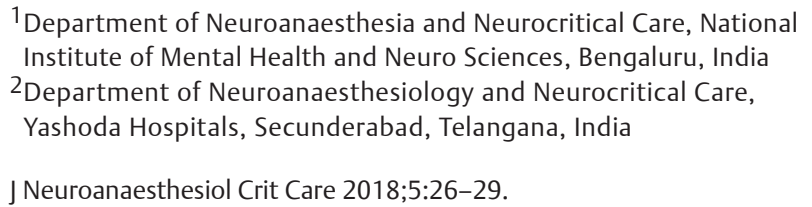

Dhritiman Chakrabarti ${ }^{1}$ Manjunatha Lakshmegowda'

\begin{abstract}
Address for correspondence Dhritiman Chakrabarti, MD, DM, Department of Neuroanaesthesia and Neurocritical Care, 3rd Floor, Faculty Block, NIMHANS, Hosur Road, Bengaluru 560 029, Karnataka, India (e-mail: dhritiman.ch@gmail.com).
\end{abstract}

\begin{abstract}
Keywords

- intraoperative seizures

- posterior fossa surgery

- tranexamic acid
\end{abstract}

We present a case series of four cerebellopontine angle surgeries with intraoperative detection seizure activity. The cases discussed follow the uniform anesthetic protocol of maintenance with infusion of propofol, fentanyl and dexmedetomidine excluding muscle relaxant, targeted to maintain bispectral index (BIS) in the range of 40 to 60 . The cases experienced either of the two scenarios: (I) Unexplained increased or widely varying BIS values associated with increases in BIS electromyography (EMG) and motor manifestation of seizure activity in limbs. (2) Varying BIS values with EMG activation of facial nerve monitoring without motor manifestation in limbs. A common theme noticed in all these cases was the utilisation of tranexamic acid in cumulative doses of 25 to $30 \mathrm{mg} / \mathrm{kg}$ and amelioration of the seizure activity with a loading dose of phenytoin $(15 \mathrm{mg} / \mathrm{kg})$. A hypothesis is being presented linking intraoperative seizures with a combination of tranexamic acid utilisation.

\section{Introduction}

Intraoperative seizures are rare occurrences due to cortical suppression by anesthetic agents. Furthermore, if intraoperative electroencephalographic (EEG) monitoring (raw or processed) is not employed, diagnosis is missed due to the use of neuromuscular blocking agents. Infratentorial tumors are rarely implicated as a cause of seizure. In this case series, we describe four patients who developed intraoperative seizures during surgery for their cerebellopontine (CP) angle tumor. In all these patients, characteristic changes were observed in the bispectral index (BIS) values which were presumed to be due to intraoperative seizure, and the BIS changes disappeared with the administration of loading dose of phenytoin intravenously. Informed written consent from the relatives of patients was obtained before writing this report.

\section{Case Reports}

\section{Case 1}

A 33-year-old male presented with a history of gait disturbance and deafness in right ear for 2 years. Magnetic resonance imaging of the brain revealed a right CP schwannoma, and microsurgical decompression was planned through retromastoid craniectomy in left lateral position. BIS electrode (BIS Quatro-sensor ${ }^{\mathrm{TM}}$, A-2000 EEG version 3.4 monitoring system, Massachusetts, USA) was placed on the forehead of contralateral side (left), and the facial nerve monitoring electrodes (NIM Response 3.0, Medtronic, Minneapolis, USA) were inserted on ipsilateral frontalis, orbicularis oculi, orbicularis oris and mentalis muscles. Patient was induced and maintained with propofol infusion using target-controlled infusion (TCI) (Fresenius Kabi, Brezins, France) with target BIS in the range of 40-60. During surgery, neuromuscular blocking agents were excluded to facilitate facial nerve electromyographic (EMG) monitoring with in-field direct electrical stimulation. Fentanyl boluses were used for analgesia. Patient was loaded with tranexamic acid intravenously at a dose of $1 \mathrm{~g}(15 \mathrm{mg} / \mathrm{kg})$ followed by $150 \mathrm{mg}(2.5 \mathrm{mg} / \mathrm{kg})$ hourly boluses throughout surgery to reduce intraoperative bleeding. During tumor dissection, the BIS, which hitherto had been stable, increased to 70 along with an increase in BISEMG ( - Fig. 1). The EEG waveform obtained from BIS monitor also showed high amplitude fast activity. The facial nerve
Dol https://doi.org/ 10.1055/s-0037-1616039. ISSN 2348-0548.
Copyright $@ 2018$ Indian Society of Neuroanaesthesiology and Critical Care
License terms

(우(1) $\Theta \circledast$ 


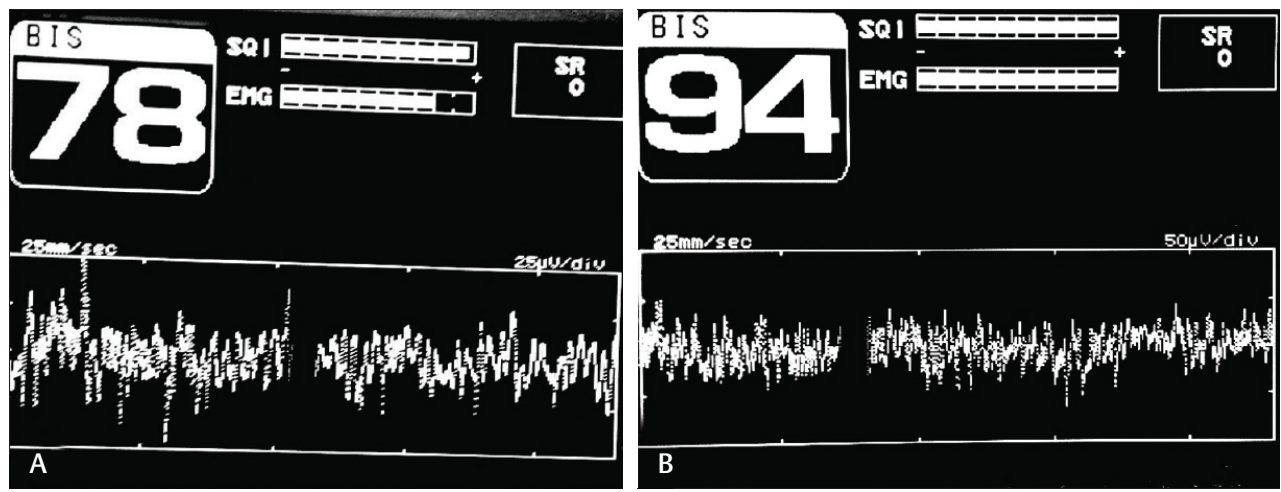

Fig. 1 High electromyographic band power in bispectral index monitor with high bispectral index value during intraoperative seizure in (A) case 2 and (B) case 4. Note the signal quality index is maintained, thus ruling out any recognized artefacts.

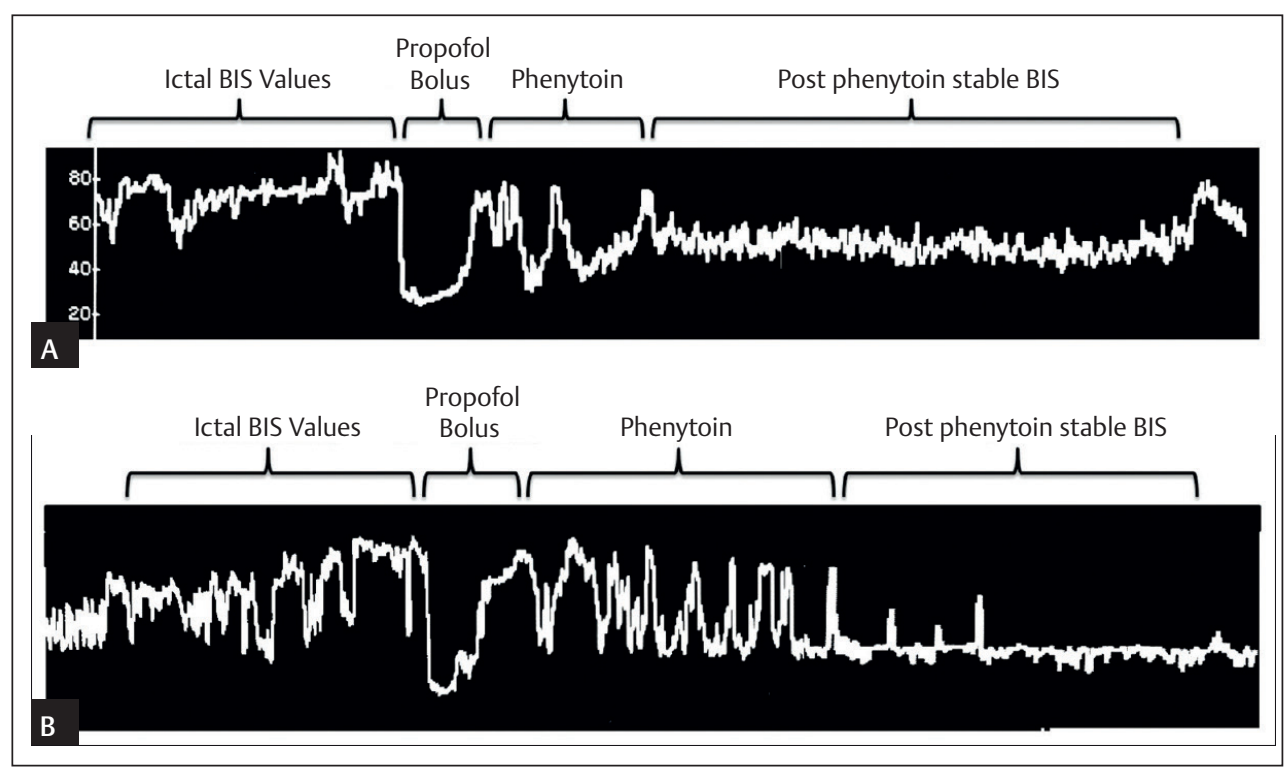

Fig. 2 Trend of bispectral index changes during intraoperative course of events. (A) Case 2 and (B) case 4.

monitor, however, did not record any EMG activity. Propofol TCI effect-site target was increased from 2.5 to $5 \mu \mathrm{g} / \mathrm{ml}$ as the increase in BIS was thought to be due to light plane of anesthesia. The EMG activity stopped transiently but resumed after $5 \mathrm{~min}$, even with TCI target of $5 \mu \mathrm{g} / \mathrm{ml}$. Later, the operating surgeon complained of jerking type of movement of the patient's neck and shoulders. Upon checking under the drapes, it was noticed that the limbs on the ipsilateral side of lesion were indeed jerking, with no movement on the opposite side. The temperature of the patient was $36.8^{\circ} \mathrm{C}$. The jerky movements and EMG activation were presumed to be motor manifestation of seizure activity, and patient was loaded with phenytoin at a dose of $15 \mathrm{mg} / \mathrm{kg}$ intravenously. After 15 min of completion of phenytoin infusion, the jerky movements and the EMG activation stopped, BIS values returned back to 40-60 range and did not recur throughout the surgery ( - Fig. 2). Recovery from anesthesia was not delayed and patient was extubated uneventfully. Postoperatively, phenytoin was not prescribed, and the patient did not have seizures at 7-day follow-up. Postoperative computed tomography scan did not reveal any causative pathology. Ipsilateral (right) seizures associated with contralateral facial twitching (BIS EMG increases) point to partial seizure with onset zone localised to contralateral (left) motor cortex although ipsilateral facial sparing (no EMG recorded in cranial nerve monitor) decreases validity of this hypothesis (due to bilateral innervation of upper face from unilateral motor cortex).

\section{Case 2}

A 45-year-old male patient underwent right $\mathrm{CP}$ angle tumor decompression under facial nerve monitoring with similar anesthetic protocol as described above. During tumor dissection, similar increases in BIS-EMG, BIS value and EEG waveform changes were noticed. However, there was simultaneous activation of the facial nerve EMG monitor (ipsilateral right) as well. After approximately 20 min of start of EMG activation, jerky movements in bilateral limbs were noted. As before, propofol bolus led to transient decrease and phenytoin administration led to permanent resolution within 20 min. Both BIS-EMG and facial nerve monitor EMG showed resolution of spontaneous activity; however, activation of in-field facial nerve stimulation was preserved ( - Figs. 1A and 2B). Bilateral seizures with bilateral facial twitching point to a generalised seizure, with an undetermined onset zone. 


\section{Cases 3 and 4}

A 32-year-old male patient underwent left CP angle schwannoma decompression under facial nerve monitoring with similar anesthetic protocol as above. Increase in BIS, BISEMG and EEG waveform changes alerted the anesthetist, whereupon TCI target was increased to $6 \mu \mathrm{g} / \mathrm{ml}$ without effect. No motor activity in limbs was noted. Spontaneous facial nerve monitoring EMG discharges commenced after $10 \mathrm{~min}$ concurrent with contralateral increase in BIS-EMG. The discharges were similar to 'popcorn' type discharges hitherto ascribed to local irritation of facial nerve. The continuous spontaneous cranial nerve monitor EMG activity prevented actual in-field stimulation-induced discharges from being identified. Thus, based on previous experience, phenytoin was administered, and predictably, the EMG activity ceased after $15 \mathrm{~min}$, with preservation of in-field stimulated EMG discharges. Isolated facial nerve territory activation suggests local irritation of facial nerve. Bilaterality of the phenomenon suggests irritation at the level of facial nucleus (where close proximity exists).

Case 4 was a 40 -year-old male patient with right CP angle schwannoma had similar intraoperative events, and phenytoin was helpful in ameliorating the EMG activity and 'popcorn' discharges (- Figs. 1B and 2B).

None of the patients had any previous history of seizure disorder. The blood glucose (measured by glucometer), serum electrolytes, temperature and arterial blood gas parameters of all three patients were within normal limits in the peri-ictal period. Scalp block had not been administered, and the dose of local anesthetics used for skin infiltration was within acceptable dose and the seizures were noticed after about 1-2 h, thus ruling out the role of excess/intravascular local anesthetics.

The said technique of propofol TCI under BIS monitoring is being followed at our institution as part of a study protocol. Until writing of this manuscript, out of $34 \mathrm{CP}$ angle surgeries conducted, with two cases of confirmed seizure activity with motor manifestations and two cases of probable seizure activity being documented, the incidence of intraoperative seizure in these surgeries seems quite high.

\section{Discussion}

Intraoperative seizure documentation is a rare event, even rarer in posterior fossa surgeries. Intraoperative BIS monitoring and avoidance of neuromuscular blockers helped in identifying the seizure episodes. Intraoperative seizure activity leading to facial muscle contraction may cause increases in BIS, which is, however, not due to EEG activation but rather increased EMG content of the signal. The EMG contamination would actually decrease the probability of documenting classical spike activity pathognomonic of seizures.

Isolated spontaneous facial territory EMG discharge (cases 3 and 4) occurs quite frequently during these surgeries and is attributed to local irritation of the facial nerve. However, in our case, the muscular contractions were bilateral (evidenced by both facial nerve EMG [ipsilateral] and BIS EMG [contralateral]). Isolated facial muscle contraction, whether due to spontaneous discharges of the facial nerve or as a part of seizure activity, was amenable to resolution with a loading dose of phenytoin. The ablation of spontaneous discharge did not affect the evoked responses of direct nerve stimulation. One possibility for these seizures could be localised depolarisation of bilateral facial nerve nuclei in the brainstem. Retrograde conduction through motor pathways to the motor cortex may cause spread of depolarisation and initiate seizure activity. ${ }^{2}$

Another factor that could have triggered this neuronal hyperexcitability was tranexamic acid administration, even though the doses used were slightly more than normal range. ${ }^{3}$ Sharma et al. had noted that, during cardiac surgeries, the median dose of tranexamic acid that resulted in postoperative convulsive seizures was $100 \mathrm{mg} / \mathrm{kg}$ with a range of 31 to $175 \mathrm{mg} / \mathrm{kg}$. ${ }^{4}$ The cumulative dose of tranexamic acid in our case series was 25 to $30 \mathrm{mg} / \mathrm{kg}$. Multiple retrospective studies have reported the incidence of postoperative seizures, attributable to tranexamic acid ranging from $0.9 \%$ to $7.3 \%$. Intraoperative seizure activity attributable to this drug, however, has not been reported. ${ }^{5}$ Tranexamic acid has been shown to inhibit gammaaminobutyric acid $\left(\mathrm{GABA}_{\mathrm{A}}\right)$-mediated inhibitory synaptic transmission and competitively inhibit glycine receptors in mice. ${ }^{5-7}$ Even with normal doses of tranexamic acid, as in our case series, neuronal handling could have resulted in spreading of localised depolarisation due to partial inhibition of GABA-mediated inhibitory mechanisms. However, this hypothesis needs to be proven.

Intraoperative seizures under general anesthesia have been reported in a variety of case reports and systematic reviews with aetiology proposed as neurosurgical procedure itself, previous history of epilepsy, cochlear implant insertion, awake craniotomy, transcranial motor evoked potential monitoring and deep hypothermic cardiac arrest. ${ }^{8-12}$ In our cases, possibly a combination of factors, namely tranexamic acid and localised neuronal irritation caused by surgery caused the seizure activity.

In the absence of neuromuscular blockade, high EMG activity contaminated the EEG recording resulting in falsely high BIS values. Sustained frontalis muscle EMG has been shown previously to cause artificially high BIS values. ${ }^{13}$ Higher BIS values during seizure activity under neuromuscular block have also been reported in previous case reports, which likely reflect hypersynchronous neuronal discharges in the frontal lobe. ${ }^{14}$ Sudden onset sustained high BIS values in the absence of known causes of EEG artefacts and under acceptable levels of anesthetic administration thus should not be rejected as artefactual, and clinical suspicion should be maintained to diagnose intraoperative seizure activity. In our cases, we could not administer muscle relaxant to eliminate confounding of the EEG signal by EMG as these events occurred during tumor dissection when facial nerve monitoring was being utilised. These patients did not have postoperative seizures, and phenytoin was not administered in the postoperative period. Hence, postoperative seizure risk did not seem to be increased with documented intraoperative seizure activity. 


\section{Conclusion}

Our findings were purely observational and serendipitous in nature but lend themselves to explanation based in previous evidence. Prospective observational studies in this group of patients are required to validate our findings. Utilisation of BIS and close observation of the EMG activity displayed are crucial to the diagnosis of this condition, and high clinical suspicion should be maintained in this context.

\section{Funding}

None.

\section{Conflict of interest}

None.

\section{References}

1 van Breemen MS, Wilms EB, Vecht CJ. Epilepsy in patients with brain tumours: Epidemiology, mechanisms, and management. Lancet Neurol 2007;6:421-430

2 Epstein CM. Epilepsy. In: Walker HK, Hall WD, Hurst JW, eds. Clinical Methods: The History, Physical, and Laboratory Examinations. 3rd ed. Ch. 56. Boston: Butterworths; 1990

3 Horrow JC, Van Riper DF, Strong MD, Grunewald KE, Parmet JL. The dose-response relationship of tranexamic acid. Anesthesiology 1995;82:383-392

4 Sharma V, Katznelson R, Jerath A, Garrido-Olivares L, Carroll J, Rao $\mathrm{V}$, et al. The association between tranexamic acid and convulsive seizures after cardiac surgery: A multivariate analysis in 11529 patients. Anaesthesia 2014;69:124-130
5 Lecker I, Wang DS, Whissell PD, Avramescu S, Mazer CD, Orser BA. Tranexamic acid-associated seizures: Causes and treatment. Ann Neurol 2016;79:18-26

6 Kratzer S, Irl H, Kochs E, Rammes G, Haseneder R. How does tranexamic acid induce seizures? Impaired inhibitory but unaffected excitatory synaptic transmission in the mouse amygdala as potential mechanism [abstract] Eur J Anaesthesiol 2013;30:4

7 Lecker I, Wang DS, Romaschin AD, Peterson M, Mazer CD, Orser BA. Tranexamic acid concentrations associated with human seizures inhibit glycine receptors. J Clin Invest 2012;122:4654-4666

8 Davis SF, Altstadt T, Flores R, Kaye A, Oremus G. Report of seizure following intraoperative monitoring of transcranial motor evoked potentials. Ochsner J 2013;13:558-560

9 Cheung AT, Weiss SJ, Kent G, Pochettino A, Bavaria JE, et al. Intraoperative seizures in cardiac surgical patients undergoing deep hypothermic circulatory arrest monitored with EEG. Anesthesiology 2001;94:1143-1147

10 Yuan Y, Peizhi Z, Xiang W, Yanhui L, Ruofei L, Shu J, et al. Intraoperative seizures and seizures outcome in patients underwent awake craniotomy. J Neurosurg Sci 2016

11 Howe J, Lu X, Thompson Z, Peterson GW, Losey TE. Intraoperative seizures during craniotomy under general anesthesia. Seizure 2016;38:23-25

12 Musser AB, Golub JS, Samy RN. Intraoperative seizure and cerebrospinal fluid leak during adult cochlear implant surgery. Cochlear Implants Int 2016;17:116-119

13 Messner M, Beese U, Romstöck J, Dinkel M, Tschaikowsky K. The bispectral index declines during neuromuscular block in fully awake persons. Anesth Analg 2003;97:488-491

$14 \mathrm{Kim} \mathrm{H}$, Kim SY. Pitfall of bispectral index during intraoperative seizure - A case report. Korean J Anesthesiol 2013;65:449-452 